\title{
Chiral Steering of Molecular Organization in the Limit of Weak Adsorbate-Substrate Interactions: Enantiopure and Racemic Tartaric Acid Domains on $\mathbf{A g}(111)$
}

\author{
Nancy M. Santagata ${ }^{\dagger}$ Amit M. Lakhani, ${ }^{\dagger}$ Bryce F. Davis, ${ }^{\dagger}$ Pengshun Luo, ${ }^{\dagger}$ \\ Marco Buongiorno Nardelli, ${ }^{\dagger,}$ and Thomas P. Pearl ${ }^{*, \dagger}$ \\ Department of Physics, North Carolina State University, Raleigh, North Carolina 27695-7518, and \\ CSMD, Oak Ridge National Laboratory, Oak Ridge, Tennessee 37831
}

Received: December 23, 2009; Revised Manuscript Received: March 4, 2010

\begin{abstract}
The influence of intermolecular interactions involving molecular chiral centers on two-dimensional organization in the limit of a weak adsorbate-surface interaction has been studied with low-temperature scanning tunneling microscopy (STM) and density functional theory (DFT). A model system composed of a chiral organic molecule, tartaric acid, and an inert metallic surface, $\operatorname{Ag}(111)$, was employed. Dual component films formed from the serial deposition of $(S, S)$ - and $(R, R)$-tartaric acid enantiomers onto this surface exhibit homochiral domain formation as revealed by molecularly resolved STM images. In contrast, a unique tartaric acid enantiomeric heteropair is experimentally and computationally verified as the basis unit of films formed via the deposition of both enantiomers simultaneously from a racemic (1:1) mixture. The molecular adsorption geometry relative to the $\operatorname{Ag}(111)$ lattice in both enantiomerically pure and racemic domains is determined primarily by the interaction of chiral centers between nearest neighbors.
\end{abstract}

\section{Introduction}

An understanding of the mechanisms that drive organization of adsorbates at surfaces is paramount in the realization of thin films with chemically and electronically tuned properties. Such two-dimensional systems are subject to two major forces that dictate the resulting overlayer structure: adsorbate-substrate and adsorbate-adsorbate interactions. In cases where the adsorbatesubstrate interaction is defined by strong chemical bonds, the formation of ordered overlayers is governed primarily by adsorption site, adsorption geometry, etc. In cases where the adsorbate-substrate interaction is weak, intermolecular forces dominate and can lead to useful and rational architectures based on intramolecular functionality.

The specific spatial arrangement of key chemical functional groups for adsorbed molecular species has been shown to lead to self-assembled, ordered, surface-bound structures for a wide variety of organic molecules. What has furthermore been demonstrated is that molecular chiral centers to which functional groups are attached are capable of steering the formation of ordered structures with stereospecificity. ${ }^{1}$ Specifically, lateral interactions between these molecules can induce two-dimensional organization and impart global chirality when confined to a surface. These studies include, but are by no means limited to, the metal-organic interfaces of enantiomers of carboxylic acids $^{2,3}$ and amino acids. ${ }^{4-6}$ For example, for the adsorption of $(R)$-alanine on $\mathrm{Cu}(100)$, it was determined that the molecular films were stabilized by hydrogen bonding between surfacebound species. ${ }^{7}$ These results indicate that molecule-induced surface chirality can be employed as a means to create directed arrangements of adsorbates on metal surfaces. All of these examples describe the chemisorption of chiral molecules onto achiral surfaces, an act that, in each case, results in organizational chirality at the global level. In strict terms, organizational

* To whom correspondence should be addressed. E-mail: tppearl@ncsu.edu.

$\uparrow$ North Carolina State University.

* Oak Ridge National Laboratory. chirality in the global sense requires that only one unique chiral domain is created and sustained over the entire surface. ${ }^{1}$ Further, the following three requirements must be met in order to sustain true global chirality: (1) intrinsically chiral modifiers (2) adsorbed to the surface in a rigid and defined geometry (3) and lending to directional and anisotropic lateral interactions. ${ }^{1}$

A natural extension of studies involving two-dimensional enantiopure films is the deposition of racemic mixtures onto surfaces. Several such studies have been carried out to date and the reported results can be generally grouped into two potential outcomes: separation of enantiomers into independent domains that retain the relative chiral structures observed as a result of independent adsorption of enantiomers, and enantiomeric mixing. For the first case, i.e., separation of isomers into enantiopure domains, a few systems have been reported to exhibit this behavior: phenylglycine/ $\mathrm{Cu}(110)^{8}$ and a biphenyl formamide/ HOPG. ${ }^{9}$ In contrast, the formation of racemic or pseudoracemic adlayers has also been observed in a fair number of cases. Recall that true racemic mixtures contain both enantiomers in equal proportions in the unit cell; a pseudoracemic layer is composed of both enantiomers, but the chirality within the adlayer is random at all length scales. Such systems include alanine/ $\mathrm{Cu}(110),{ }^{10,11}$ alanine/ $\mathrm{Cu}(100),{ }^{10}$ a phenyl benzoate/HOPG (liquid/solid), ${ }^{12}$ 9,10-diiodooctadecan-1-ol/ HOPG ${ }^{13}$ and 9,10-diiodooctadecanoic acid/HOPG ${ }^{14}$ In fact, the unit cells of alanine/Cu(100), ${ }^{10} 9,10$-diiodooctadecan-1ol/HOPG,$^{13}$ and 9,10-diiodooctadecanoic acid/HOPG ${ }^{14}$ consist of both enantiomers in a 1:1 ratio. 6-Nitrospiropyran/Au(111) is an interesting system, wherein two-dimensional adlayer domains formed from a racemic mixture are composed of one-dimensional homochiral chains. ${ }^{15}$ Similarly, the formation of homochiral cysteine pairs on $\mathrm{Au}(110)$ has been reported upon annealing the racemic agglomerates. ${ }^{5}$ Finally, prochiral dicarboxystilbene forms both homochiral and racemic (1:1) domains on $\mathrm{Cu}(110){ }^{16}$

Presented in this work is the study of a classic chiral molecule, tartaric acid $\left(\mathrm{C}_{4} \mathrm{O}_{6} \mathrm{H}_{6}\right)$, adsorbed on $\mathrm{Ag}(111)$ in the form of both 
enantiopure monolayers as well as binary films of enantiomers resulting in locally racemic domains. It has been shown experimentally that chiral domains of this molecule form upon chemisorption of enantiopure forms of the tartrate on the (111) and (110) crystal planes of $\mathrm{Cu}$ and $\mathrm{Ni}^{2,3,17,18}$ Density functional theory (DFT) calculations and kinetic Monte Carlo simulations confirm these results, ${ }^{19,20}$ and very recently determined the reaction mechanism. ${ }^{21}$ In other words, films composed of pure $(S, S)$ - or $(R, R)$-tartaric acid represent nonsuperimposable mirror images of each other, just as the enantiomerically pure solids. The logical conclusion, then, is that the internal chiral centers must control, to some extent, the overall organization of the film. Considering that constituents that are attached to the chiral centers are not involved in bonding to the surface, global film chirality must then be controlled by intermolecular hydrogen bonding at chiral centers. Notably, in contrast to the relevant tartaric acid literature, it was proposed that intramolecular hydrogen bonding contributed to the stability of the bitartrate adsorbed on $\mathrm{Cu}(110) .^{20}$

To date, just two instances of adsorption of tartaric acid from a racemic mixture have been reported in the literature. ${ }^{22,23}$ Low energy electron diffraction (LEED) results indicate that domain organization on $\mathrm{Cu}(110)$ is highly coverage dependent: at low coverages, the films separate into enantiomorphous domains of bitartrate species, and at higher coverages a racemic lattice that is composed of monotartrate adsorbates is proposed..$^{23}$ This separation of enantiomers into homochiral bitartrate domains upon adsorption of racemic tartaric acid onto $\mathrm{Cu}(110)$ at low coverage was confirmed recently with scanning tunneling microscopy. ${ }^{22}$ Both studies indicate that a strong molecule-surface interaction precludes the formation of racemic overlayers despite the natural affinity between opposite handed enantiomers. ${ }^{24,25}$

In contrast to the chemisorption to $\mathrm{Cu}$ and $\mathrm{Ni}$ surfaces, tartaric acid enantiomers are only weakly bound to $\operatorname{Ag}(111) .{ }^{26}$ It has been shown that tartaric acid enantiomers on $\mathrm{Ag}(111)$ experience intermolecular interactions that give rise to a sufficiently rigid adsorption geometry, allowing for the realization of global organizational chirality in a similar fashion to what has been observed for molecules with a strong interaction with the underlying surface. ${ }^{26-28}$ The goal of the present study is to determine the exact influence of molecular chirality on twodimensional organization in the absence of strong chemical bonds to the surface. To further understand the stability and formation mechanism of the single component, enantiomerically pure domains, detailed low-temperature scanning tunneling microscopy (STM) studies were performed regarding the concurrent adsorption of both $(S, S)$ - and $(R, R)$-tartaric acid enantiomers onto $\mathrm{Ag}(111)$. Presented is a comparison of mixed enantiomer films formed via two different deposition methods: serial deposition of enantiomers versus simultaneous deposition from a racemic mixture. It will be shown that serial deposition results in the formation of homochiral domains that adopt the same two-dimensional structure as the enantiopure films. In contrast, films formed from a racemic mixture of tartaric acid enantiomers do not laterally separate into homochiral domains under the deposition conditions considered. Each type of film is composed of unique fundamental molecular units, the identity of which will be addressed in terms of a new, enantiomeric heteropair basis for the racemic films. Gas-phase density functional theory computations support a $(R, R) /(S, S)$ heteropair as the basis for the racemic films. The formation of the two different types of domains will be discussed in terms of a stereochemically driven mechanism that defines not only lateral interactions, but also molecular orientation relative to the surface.
These results indicate that internal molecular structure, namely chirality, can be utilized in the realization of directed twodimensional organization.

\section{Experimental and Computational Methods}

All experiments were carried out in an ultrahigh vacuum (UHV) system (base pressure $7.5 \times 10^{-11}$ Torr) that is described in great detail elsewhere. ${ }^{29}$ Briefly, the UHV chamber is equipped with a custom-built variable and low-temperature scanning tunneling microscope (STM), a high energy ion gun (Omicron NanoTechnology $\mathrm{GmbH}$ ) and electron bombardment heater for sample preparation, and low energy electron diffraction (LEED) optics (Omicron NanoTechnology $\mathrm{GmbH}$ ) for sample characterization. The $\mathrm{Ag}(111)$ single crystal (MaTecK, $\mathrm{GmbH})$ was prepared by noble gas ion sputtering $\left(\mathrm{Ar}^{+}, 1 \mathrm{keV}\right.$, $I_{\text {sputter }} \approx 3.5 \mu \mathrm{A} / \mathrm{cm}^{2}$ ) followed by annealing at $800 \mathrm{~K}$. STM and LEED routinely confirmed the preparation of a clean, wellordered $\operatorname{Ag}(111)$ surface. Solid $(R, R)-,(S, S)$-, and DL-tartaric acid (enantiomerically pure: Fluka, $\geq 99.5 \%, \geq 99.0 \%$; racemic: Aldrich, 99\%) crystals were used as received and without further purification. Differentially pumped glass vials (MDC Vacuum Products) separated from the main UHV chamber by gate valves were used for line of sight, solid-source dosing. These sources were mounted in two positions on the UHV chamber, allowing for sequential dosing as described herein, with the mouth of each doser mounted approximately 8 in. away from the sample surface. All forms of tartaric acid were degassed at $385 \mathrm{~K}$ for several hours prior to dosing, and exposure to the $\operatorname{Ag}(111)$ was carried out only after the crystal cooled to $\sim 320 \mathrm{~K}$. Typical exposure times of $150 \mathrm{~s}$ for the tartaric acid solid heated to 385 $\mathrm{K}$ (resultant background pressure during dosing: $\mathrm{P}_{\mathrm{UHV}} \sim 2 \times$ $10^{-9}$ Torr) resulted in surface coverages of $<1$ monolayer (ML) as determined by STM.

All STM images presented herein were obtained at room (300 $\mathrm{K})$ or low $(83$ and $17 \mathrm{~K})$ temperature under constant current conditions and with the sample biased relative to the tip. Mechanically cut $90 \% \mathrm{Pt} / 10 \%$ Ir tips were prepared in situ on a bare metal surface via controlled tip crashes, short voltage pulses in tunneling $( \pm 5 \mathrm{~V})$, and high voltage $(+200 \mathrm{~V})$ field emission junctions. Image processing was performed with WSxM, a free application developed by Nanotec Electronica S.L. ${ }^{30}$

Plane-wave (periodic boundary) calculations were carried out in the DFT code PWscf, ${ }^{31}$ using the Perdew-Burke-Ernzerhof (PBE) exchange-correlation functional as implemented in the generalized gradient approximation (GGA) and RRKJ-type ultrasoft pseudopotentials. The plane-wave kinetic energy and charge density cutoffs for all calculations were 36 and 288 Ryd, respectively. All parameters (i.e., cutoff energy, $k$-points, vacuum spacing) were chosen such that they were individually converged to $1 \mathrm{meV} / \mathrm{atom}$. Structures were considered fully relaxed when the interatomic forces on all atoms were reduced to $0.01 \mathrm{eV} / \AA$ or less.

Independent optimization of the structures of both the clean $\operatorname{Ag}(111)$ slab and the gas-phase tartaric acid molecule were completed prior to performing the molecule/surface and molecule/ molecule interaction studies. Prior to construction of the slab, bulk Ag parametrization (i.e., cutoff energy and $k$-points) yielded a lattice parameter of $4.21 \AA$. The clean $\mathrm{Ag}(111)$ surface was modeled by using a 64 atom slab, which contained four atomic layers composed of 16 atoms per layer. A $6 \times 6 \times 1$ Monkhorst-Pack $k$-point mesh generation scheme yielded a set of 20 symmetry-irreducible $k$-points. The clean $\operatorname{Ag}(111)$ supercell utilized $20 \AA$ of vacuum space in the $Z$ direction to 
accommodate tartaric acid molecules in a standing up geometry. The positions of all $\mathrm{Ag}$ atoms in the bottom two layers of the slab were fixed at the calculated bulk positions, while the positions of the atoms in the top two layers were allowed to relax freely. Geometry optimization of the slab resulted in a compression of the top two layers in the $Z$ direction to experimentally measured values. ${ }^{32}$

Relaxation of isolated gas-phase $(S, S)$ - and $(R, R)$-tartaric acid molecules were carried out with a single $k$-point at the high symmetry $\Gamma$ point. Structural optimizations of single enantiomers resulted in geometries that were consistent with published experimental $^{33,34}$ and theoretical ${ }^{35}$ results. The molecules are stabilized by an intramolecular hydrogen bond that forms between the $\mathrm{O}$ atom of the terminal carboxylic acid group and the $\mathrm{H}$ atom of the internal hydroxyl group attached to the adjacent chiral center. Two of these interactions occur within a single molecule, and the planar five-membered rings that form at the termini $\left(\mathrm{C}_{1}-\mathrm{O}-\mathrm{H} \cdots \mathrm{O}-\mathrm{C}_{2}\right.$ and $\left.\mathrm{C}_{4}-\mathrm{O}-\mathrm{H} \cdots \mathrm{O}-\mathrm{C}_{3}\right)$ adopt a trans conformation with respect to one another.

All images of computational results presented herein were prepared with use of XCrySDen, a free application developed by Anton Kokalj. ${ }^{36}$

\section{Results and Discussion}

Experiments were carried out in two parts and what follows is a comparison of dual component films that were formed from two different growth procedures. The first type of mixed enantiomer film was grown by what will be referred to as serial exposure: clean $\operatorname{Ag}(111)$ was first exposed to $(S, S)$-tartaric acid, then, upon cessation of the $(S, S)$-tartaric acid flux, the resulting surface was exposed to a dose of $(R, R)$-tartaric acid. The second type of dual component film was deposited via an alternate method: exposure of the clean $\mathrm{Ag}(111)$ crystal to a racemic (50: 50) mixture of the two enantiomers. Herein, films grown by the former procedure are called serial films, and those formed by the latter are referred to as racemic films.

Low-Temperature Scanning Tunneling Microscopy. Previous LEED and room temperature STM results showed that enantiopure films of tartaric acid are weakly bound to the $\operatorname{Ag}(111)$ substrate. ${ }^{26}$ Room temperature STM studies for the racemic films confirm the same behavior, namely mobile domains whose perimeters are easily perturbed by repeated scanning of the STM tip. Therefore, in an effort to elucidate the molecular-level structure in the absence of thermally induced motion, the dual-component tartaric acid monolayers were imaged at cryogenic temperatures. Figure 1 displays lowtemperature $(83 \mathrm{~K})$ topographic STM images that provide a direct comparison between the two different types of $(S, S)$-/ $(R, R)$-tartaric acid films formed from the two different codeposition procedures. It is observed that films formed from serial deposition (Figure 1a) are composed of homochiral domains that adopt the same organizational structure as the single component films. The relative chirality of the $(S, S)$ - and $(R, R)$ tartaric acid domains is evidenced by structural modulation bands that arise for the enantiopure films. ${ }^{26}$ These features, whose directionality are enantiomer dependent and, therefore, are a signature of a specific chiral arrangement for this system, are the result of a mismatch between the tartaric acid and the $\mathrm{Ag}(111)$ lattice and the spacings between the bands are sensitive to the local molecular density. The bright area between the two enantiopure domains is most likely the result of domain growth overlap, but the presence of separate chiral domains indicates that the formation of a single tartaric acid layer is preferred to direct multilayer growth.
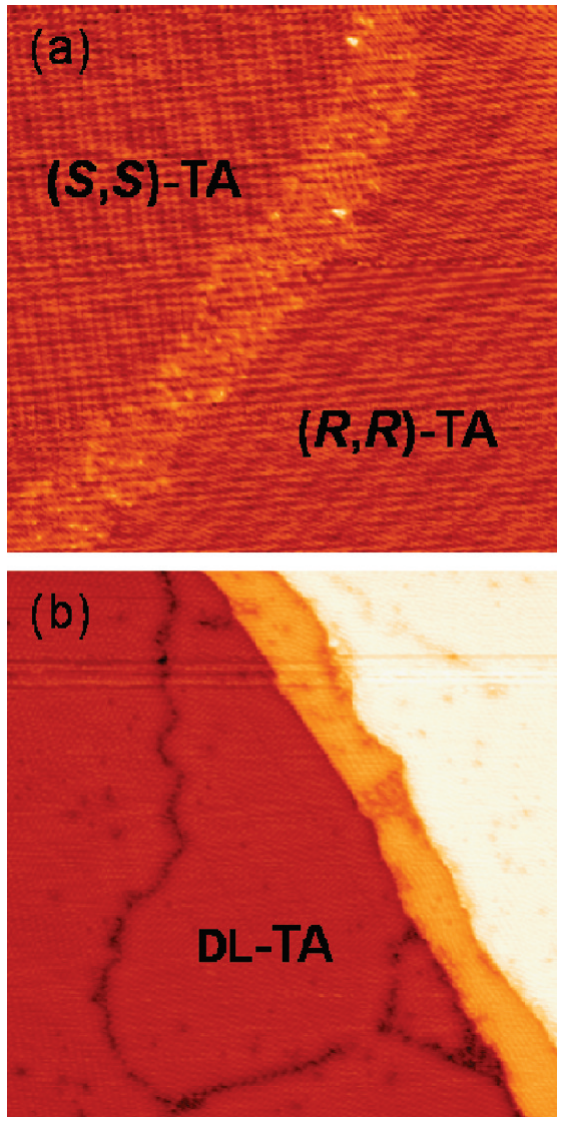

Figure 1. Low-temperature $\left(T_{\text {sample }}=83 \mathrm{~K}\right)$ STM images of longrange structures formed from codeposition of tartaric acid (TA) enantiomers onto $\operatorname{Ag}(111)$. The enantiospecific domains (a) were generated via serial exposure from separate $(S, S)$ - and $(R, R)$-tartaric acid sources, while the racemic domain (b) was generated via a simultaneous exposure from a racemic $(1: 1)(S, S)-/(R, R)$-tartaric acid mixture. Image areas: $850 \AA \times 850 \AA$. Tunneling conditions: $I_{\mathrm{t}}=100$ $\mathrm{pA}, V_{\text {sample }}=127$ (a) and $50 \mathrm{mV}$ (b).

In contrast to the serially grown mixed enantiomer film, it is clear that the racemic monolayer does not exhibit the same structural features (Figure 1b). In addition to the observation of numerous vacancy sites (small depressions), multiple small domains exist whose randomly oriented boundaries often terminate at or near $\operatorname{Ag}(111)$ step edges. Large domains are only observed on large terraces away from silver step edges, though the vacancy sites persist throughout the film. It is unlikely that these vacancy sites are related to defects in the underlying $\mathrm{Ag}(111)$ substrate because the defect density within the adsorbate film is far greater than that observed for the clean $\operatorname{Ag}(111)$ surface. No such irregularities are observed within the enantiopure domains, which are in fact defect free for several tens of nanometers. Furthermore, a weak molecule-surface interaction and lack of registry between the tartaric acid films and the underlying metal lattice preclude any such relation for the two types of defects.

A prominent difference between the two films (i.e., serial versus racemic) lies in the respective structural modulation bands. Figure 2 is a comparison between an enantiopure domain of $(S, S)$-tartaric acid (Figure 2a) and one of DL-tartaric acid (Figure 2b). The presence of identical modulation bands in the serial films indicates that the adsorbate structure is the same as that for the enantiopure depositions, so for simplicity only a single component enantiopure film will be considered here. The serial films exhibit parallel bands of consistent spacing and alignment that are present throughout the entirety of the film 

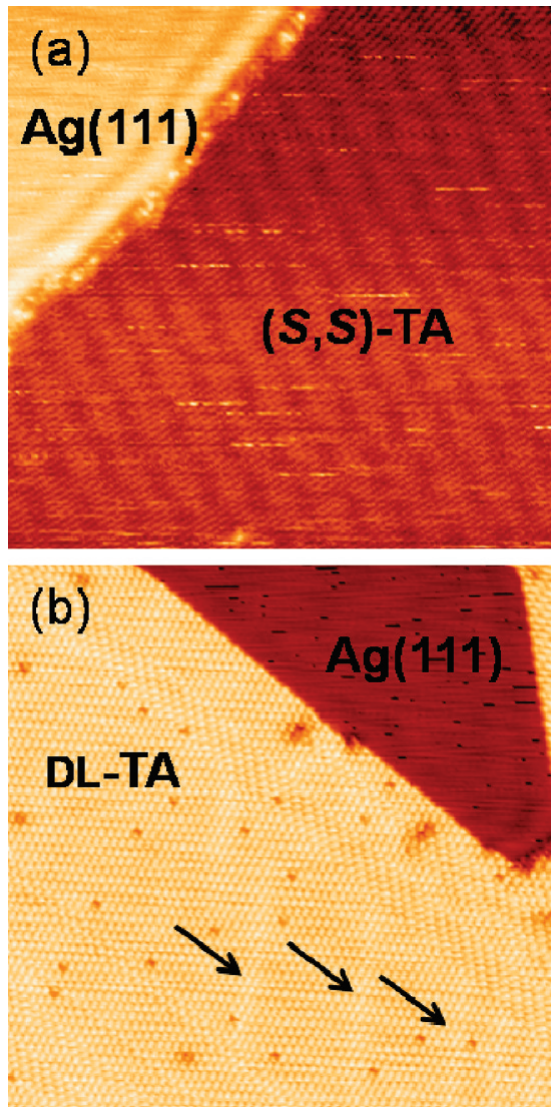

Figure 2. Low-temperature $\left(T_{\text {sample }}=83 \mathrm{~K}\right)$ STM images of organizational structures formed from deposition of enantiomerically pure $(S, S)$-tartaric acid and racemic tartaric acid (TA) (a and b, respectively) on $\operatorname{Ag}(111)$. The unique structure modulations in the racemic film are highlighted by arrows (b). Image areas: $600 \AA \times 600$ $\AA$. Tunneling conditions: $I_{\mathrm{t}}=100 \mathrm{pA}, V_{\text {sample }}=25$ (a) and $56 \mathrm{mV}$ (b).

(43 A for this particular domain); however, the racemic domains do not appear to exhibit the same long-range structural order. Referring specifically to the bright, near vertical features identified by arrows in Figure 2b, the spacing between adjacent bands here is aperiodic: from left to right, the spacings are 118, 104, and $42 \AA$. Following the conclusion that the modulation bands of the pure films are structural in nature (a result of changes in local packing density as opposed to standing wave patterns generated from the $\operatorname{Ag}(111)$ surface state scattering), ${ }^{26}$ the differences in modulation features between the two films must also be due to inherently unique structural assemblies.

In addition to differences in long-range ordering of the two types of monolayers, there is a distinct topographic contrast relative to the clean $\operatorname{Ag}(111)$ terraces that is specific to the composition of the domains. The racemic films appear as a topographic protrusion $(\Delta h=1.07 \pm 0.10 \AA)$ relative to the clean $\operatorname{Ag}(111)$ surface (Figure 2b) while the enantiopure films image as depressions relative to the Ag terraces (Figure 2a, $\Delta h=-0.30 \pm 0.02 \AA$ ). Because STM is a convolution of structural and electronic features for a given system, it is clear that domain specific contributions to the local density of states lead to differences in the STM topographic contrast. ${ }^{37}$

Figure 3 displays high-resolution low-temperature STM images of the molecular level arrangement of the two types of mixed enantiomer films. Measurements of molecular features quoted throughout are the result of statistical analysis of multiple experimental runs. Each bright feature in the enantiopure films (Figure 3a,b) corresponds to a single tartaric acid molecule, e.g.,
$6.05 \pm 0.09 \AA \times 3.55 \pm 0.07 \AA$ for the $(S, S)$-tartaric acid case. The molecules adopt one of two orientations relative to each neighboring molecule, namely end-to-end or end-to-side, leading to two different absolute orientations on the surface. This arrangement results in an enantiopure unit cell with a $p 2$ surface space group, the average dimensions of which are corroborated by LEED measurements. ${ }^{26}$ The $(R, R)$ - and $(S, S)$-tartaric acid/ $\mathrm{Ag}(111)$ unit cells are mirror images of one another, and serve as further evidence that internal molecular chirality is preserved upon adsorption to the $\mathrm{Ag}(111)$ surface.

Though each image corresponds to an identical scan size, only five unit cell widths are observed for the $(S, S)$-tartaric acid (Figure 3a), while six unit cell widths are observed for the $(R, R)$ tartaric acid (Figure $3 b$ ). In this instance, the packing density of the $(R, R)$-tartaric acid film is greater than that of the $(S, S)$ tartaric acid and is such that individual molecules cannot be resolved along the entire unit cell. This variation in local coverage supports the assertion of a weakly bound adsorbate that does not exhibit a preference toward any single binding site with respect to the underlying $\operatorname{Ag}(111)$ lattice.

The orientation of the tartaric acid molecules relative to the $\operatorname{Ag}(111)$ substrate can be deduced from the high-resolution topographic STM data. The size and shape of the features measured from the data indicates that the molecules interact with the silver surface such that the plane defined by the fourcarbon backbone is parallel to the plane of the underlying surface. Additionally, because molecular chirality is transferred to global film chirality, intermolecular interactions must involve at least one chiral center. This in turn leads to the assertion that the internal hydroxyl groups must be oriented away from the surface and toward the vacuum such that they are available for the formation of lateral intermolecular hydrogen bonds. A topdown view of the proposed adsorption geometry for both the enantiopure $(S, S)$ - and $(R, R)$-tartaric acid domains is depicted in Figure 3c. A potential $\mathrm{O}-\mathrm{H} \cdots \mathrm{O}$ interaction is highlighted for both enantiopure films. Note that this image is presented for diagrammatic purposes only and does not represent the result of density functional theory simulations.

A high-resolution STM image of the film that was grown by sublimation of the racemic mixture is presented in Figure 3d. At this length scale, it becomes increasingly obvious that the molecular level structure does not correspond to that observed after deposition of the enantiopure molecules. Instead, each bright feature appears to be a pair of tartaric acid molecules. Two experimental observations support this conclusion: first, each feature (10.15 $\pm 0.11 \AA$ long) appears to be comprised of two smaller features $(5.08 \pm 0.11 \AA$ diameter $)$ and, second, smaller apparently unpaired features $(5.66 \pm 0.15 \AA$ diameter) exist at the periphery of the domains. These monomers are most likely the result of either slight enantiomeric excess in the sublimated racemic tartaric acid crystals or, more likely, an imbalance or variation in the local distribution of enantiomers.

A side-on view of the proposed adsorption geometry for the pairs of features observed in these films is depicted in Figure 3e. In contrast to the molecular geometry observed in the enantiopure films, for racemic tartaric acid films the plane of the molecule defined by the carbon backbone is oriented perpendicular to the plane of the surface. Again, this image does not represent the result of DFT simulations but rather is presented to illustrate the orientation of the tartaric acid molecules with respect to the $\operatorname{Ag}(111)$ surface that is inferred from the experimental evidence.

It is observed that the paired molecular basis for the racemic films adsorbs in such a way that numerous relative orientations 

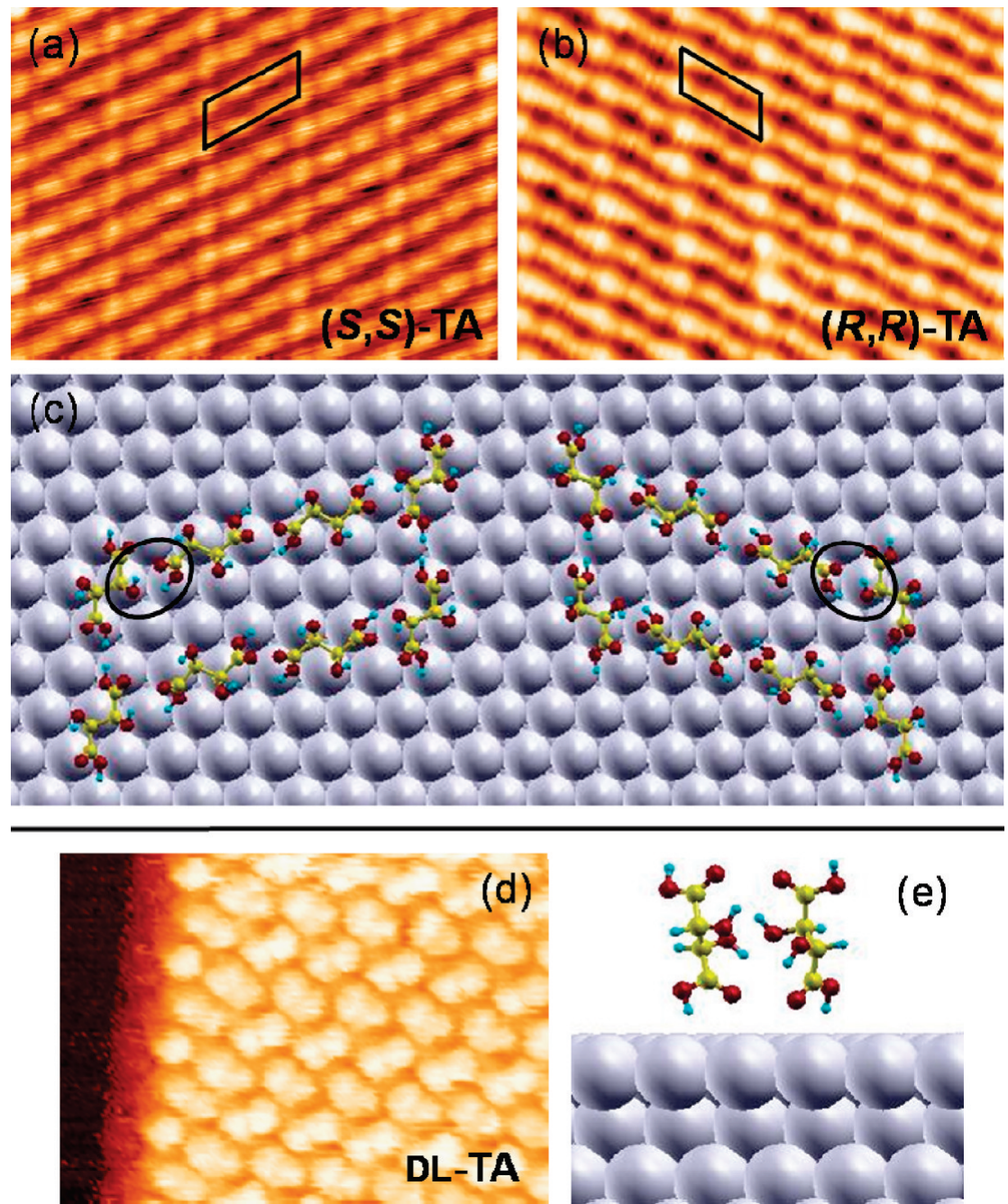

(e)

Figure 3. STM images of molecular level arrangement of enantiomerically pure (a and b) and racemic (d) domains on Ag(111). Unit cells are drawn for each enantiopure film. Structural models are also presented for the proposed enantiopure (c) and racemic (e) geometries. Intermolecular hydrogen bond interactions involving chiral centers are highlighted in panel c. Image areas: $65 \AA \times 90 \AA$. Tunneling conditions: $I_{\mathrm{t}}=100 \mathrm{pA}$, $V_{\text {sample }}=46$ (a) and $50 \mathrm{mV}$ (b and c). $T_{\text {sample }}=83$ (a and c) and $17 \mathrm{~K}(\mathrm{~b}$ ).

are possible, allowing for the creation of several different domain ordering schemes in addition to the one described in Figure 3c. Similar observations were made for a series of two-dimensional crystals of anthrone $\left(\mathrm{C}_{14} \mathrm{H}_{10} \mathrm{O}\right)$ derivatives physisorbed onto a graphite surface. ${ }^{38}$ Figure 4 depicts three such unique cases observed concurrently in the racemic tartaric acid films that are the result of competing interactions between new paired basis units. In the first case (Figure 4a), three different crystalline domains are shown that order in one of two ways. The smaller domains on the right side of the image are composed of perfectly aligned features, while the larger domain on the left is not. Aside from an apparent shift corresponding to one-half of the unit cell, the boundary between the uniform domains is nearly perfect, while the boundary between the ordered and "disordered" domains lacks an organized structure. The area enclosed by the small box is enlarged in Figure $4 \mathrm{~b}$ and highlights further evidence for enantiopairing: the small vacancies observed within the uniform domains appear to be composed of a single molecular feature and a single vacancy site.

Figure $4 \mathrm{c}$ depicts another frequently observed pattern composed of regions where the orientation of neighboring features changes very frequently, creating a herringbone-like pattern. The boundary between the two visible domains again lacks any discernible order. Figure 4d displays the third kind of domain observed, namely a region composed mainly of one type of feature (similar to that shown in Figure 4b), but containing mirror planes running diagonally across the image. Considering that each of the bright features is a molecular pair, i.e., a tartaric acid dimer, it is possible that the relative orientation of the two molecules can be reversed along the mirror plane, creating a corresponding reversal of the unit cell and, therefore, of the overall domain organization. However, this reversal is not observed to persist for more than a few unit cells (in the direction perpendicular to the mirror plane) before a similar mirror plane is encountered, resulting in a net bulk organization of a single direction. Similar results have been reported for the adsorption of heptahelicene $\left(\mathrm{C}_{30} \mathrm{H}_{18}\right)$ molecules on $\mathrm{Cu}(111) .{ }^{39}$

Although the enantiospecific identity of the tartaric acid pairs cannot be resolved with STM topographic imaging, it can be inferred that the unique basis unit is composed of both an $(S, S)$ and an $(R, R)$-tartaric acid molecule. Bulk racemic tartaric acid is a true racemic compound: in the three-dimensional solid, both enantiomers are present in a 1:1 ratio in the unit cell. ${ }^{24}$ Such a stoichiometry indicates that a single enantiomer possesses a greater affinity for the opposite enantiomer than for the identical one. In fact, this is true for $90-95 \%$ of all racemic compounds. ${ }^{25}$ It follows that when confined to two dimensions, as on a surface, for example, the interaction between opposite enantiomers will dominate and the film will be composed of enantioheteropairs. Additionally, separation into homochiral conglomerates is not 

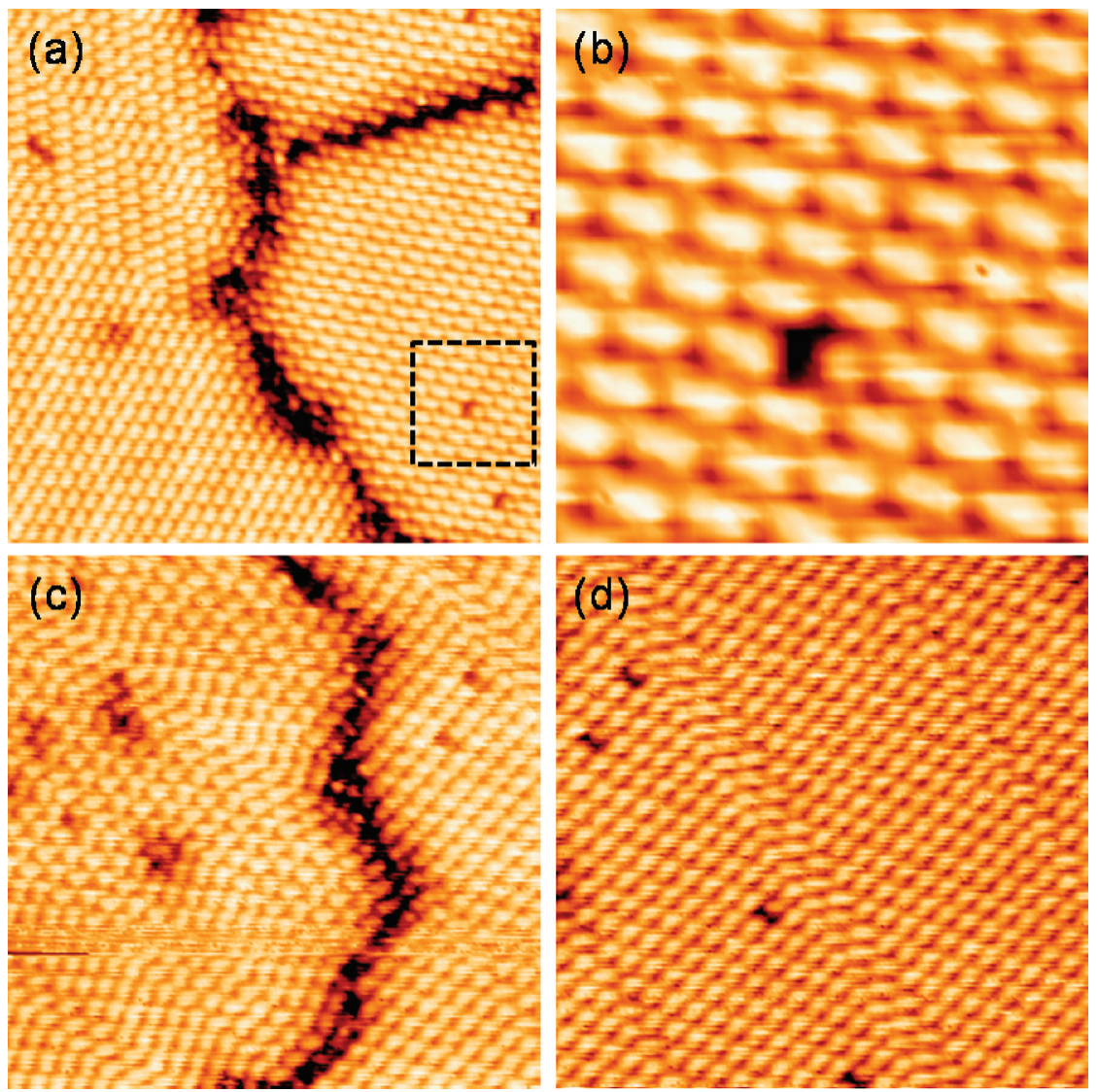

Figure 4. Low-temperature $\left(T_{\text {sample }}=83 \mathrm{~K}\right)$ STM images of the different molecular level arrangements observed over the course of the racemic tartaric acid/ $\mathrm{Ag}(111)$ experiments. The area enclosed by the box in panel a is enlarged in panel b. Image areas: $275 \AA \times 275 \AA$ (a), $70 \AA \times 70 \AA$ (b), $230 \AA \times 230 \AA$ (c), and $215 \AA \times 215 \AA$ (d). Tunneling conditions: $I_{\mathrm{t}}=100 \mathrm{pA}, V_{\text {sample }}=50 \mathrm{mV}$.

observed upon adsorption of racemic tartaric acid onto $\mathrm{Ag}(111)$ and this fact serves as an indication that a novel basis unit is the cause. Despite reports in the literature of enantioseparation, ${ }^{8,9}$ the formation of racemic or pseudoracemic adlayers has also been observed in several cases. ${ }^{10-14,40}$ In fact, the unit cells of alanine/Cu(100), ${ }^{41}$ 9,10-diiodooctadecan-1-ol/HOPG, ${ }^{13}$ and 9,10diiodooctadecanoic acid/HOPG ${ }^{14}$ consist of both enantiomers in a 1:1 ratio. The lack of long-range order for the racemic film, in comparison to what has been observed for enantiopure tartaric acid on $\operatorname{Ag}(111)$, indicates that there is relatively weak interaction between the tartaric acid pairs as well as with the underlying $\operatorname{Ag}(111)$ surface. For the enantiopure tartaric acid films, the chiral centers steer the formation of an organized structure along two major directions resulting in a hydrogen bonded network with stereospecificity. In contrast to this, the chiral centers in the individual tartaric acid molecules for the racemic film direct the formation heteropair dimers but not extended order on the surface. To further support the conclusions regarding molecular orientation and relative strength of interadsorbate interactions, analysis of an enantiopure, $(S, S)$-tartaric acid film with the growth of a second molecular layer is presented in the Supporting Information.

Density Functional Theory Modeling. Enantiopure Tartaric Acid Adsorption Geometry. In an effort to confirm the adsorbate geometry of enantiopure tartaric acid films deduced from STM studies, density functional theory (DFT) was used to model single, isolated tartaric acid adsorbates on a $\operatorname{Ag}(111)$ surface. Results describing potential adsorption geometries of single $(S, S)$-tartaric acid enantiomers on $\mathrm{Ag}(111)$ are summarized in Figure 5. The corresponding data for $(R, R)$-tartaric acid, which show the same trend, are shown in the Supporting Information.
Although the molecule is believed to be oriented with its molecular axis parallel to the surface and with the internal $\mathrm{OH}$ groups pointing toward the vacuum, four different potential geometries were considered. The naming convention presented here is based upon both the orientation of the molecular axis relative to the plane of the surface and the orientation of the chiral $\mathrm{OH}$ groups with respect to the surface. For the first geometry, $\mathrm{SS} 1$, the internal $\mathrm{OH}$ groups lie in a single $X Z$ plane (a plane that is perpendicular to the plane of the surface). The second geometry, SS2, is a "standing up" geometry, whereby the interaction between the molecule and the surface occurs through one of the terminal carboxylic acid groups. The final two geometries consider both the four-carbon backbone and the internal hydroxyl groups in an $X Y$ plane parallel to the plane of the surface but differ in the interaction with the surface: SS3 lies with the $\mathrm{OH}$ groups oriented toward the surface and SS4 lies with the $\mathrm{OH}$ groups oriented away from the surface (toward the vacuum). It follows that SS4 corresponds to the geometry assignment deduced from the experimental results. Because the molecule-surface interaction is believed to be very weak, initial geometries (i.e., geometries prior to relaxation) were chosen such that the molecule was centered in the $X Y$ plane of the supercell, rather than placing the molecule at or near a specific adsorption site. In each of the four cases, the initial distance between the topmost silver layer and the nearest atom of the tartaric acid molecule was approximately $2 \AA$. The molecules were centered in the supercells such that the distance between the respective centers of mass was $12 \AA$, resulting in a minimum separation between nearest atoms of isolated TA adsorbates in neighboring supercells of 6 (SS1) to $9 \AA$ (SS2). 


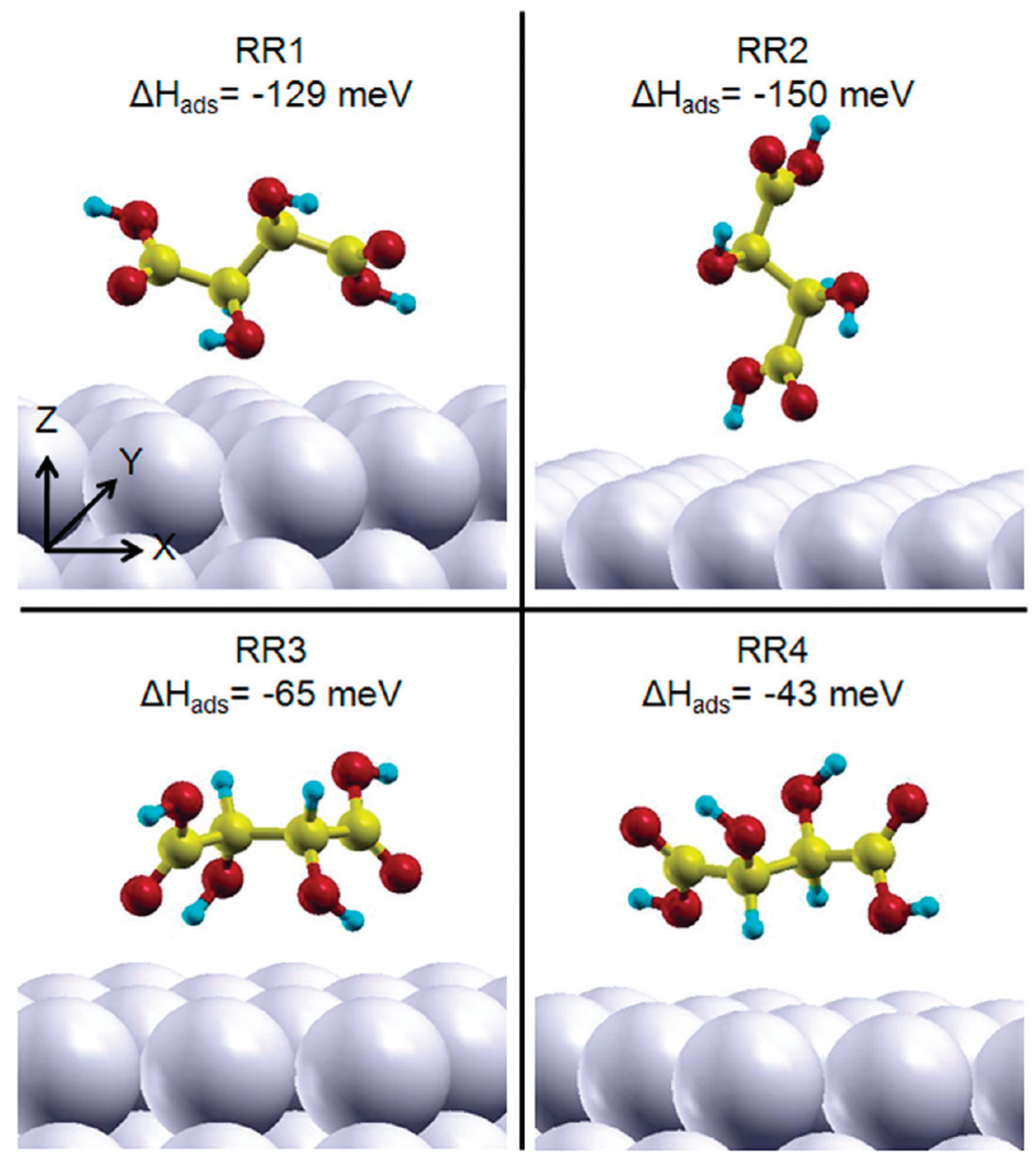

Figure 5. Density functional theory results for four potential adsorption geometries for a single $(S, S)$-tartaric acid enantiomer on a $\mathrm{Ag}(111)$ slab. Atomic colors: Ag (silver), C (yellow), $\mathrm{O}$ (red), and $\mathrm{H}$ (blue).

Figure 5 shows the results for the four previously described $(S, S)$-tartaric acid geometries, along with the final adsorption enthalpies of the relaxed structures. In each case, upon relaxation the molecule moved away from the surface and toward the vacuum, increasing the molecule-surface distance to between 2.8 and $3.5 \AA$ (where the final $\mathrm{Ag}$-tartaric acid separation is again measured between the topmost silver layer and the nearest atom of the molecule). The molecules, in general, retained their gas-phase geometry, whereby the lone $\mathrm{O}$ atom of the terminal carboxylic acid group and the $\mathrm{OH}$ group on the adjacent chiral carbon form a five-membered ring through an $\mathrm{O}-\mathrm{H} \cdots \mathrm{O}$ interaction. The planes containing the two five-membered rings retained their trans configuration with respect to one another, although the angle changed slightly over the course of the relaxation, presumably to minimize the atomic forces that occur upon interaction with the $\operatorname{Ag}(111)$ surface.

The adsorption enthalpies $\left(\Delta H_{\text {ads }}\right)$ indicate that the most energetically favorable structure in both cases is the standing up geometry, a result that does not corroborate the conclusions made from the experimental data. Although accurate structures are routinely predicted for strong-binding systems (i.e., chemisorption), it is well-known that approximate-functional DFT is unable to correctly reproduce weak van der Waals interactions (i.e., induced dipole-dipole interactions, $\sim 0.1 \mathrm{eV}$ or less). ${ }^{42}$ Therefore, the differences in calculated enthalpies between any two of these structures, due to their small magnitudes, cannot be considered accurate $e^{43,44}$ or trustworthy and no concrete conclusions can be made regarding preferential adsorption geometries for the tartaric acid/Ag(111) system. The results are valuable, however, as they reinforce the assertion of a decidedly small molecule-surface interaction. Because the structure of the films must in turn be dictated by intermolecular hydrogen bonding (i.e., permanent dipole-dipole interactions), which is significantly stronger $(\geq 0.1 \mathrm{eV})$ than van der Waals interactions, we postulate that in the limit of domain growth and interaction with nearest-neighbors, the lowest energy configuration for the molecule would change in order to maximize hydrogen bonding interactions between chiral centers.

Racemic Tartaric Acid Pair Identity. Gas-phase pair identity density functional theory simulations were carried out in an attempt to identify the composition of the paired features observed in the racemic tartaric acid films. The pair calculations that are presented in this section aim to predict interaction energies that are based upon much stronger forces (hydrogen bonds) than those discussed in the previous section (van der Waals forces), therefore the study was undertaken despite the previous results. For these gas-phase pair calculations, the distance between the closest atoms in neighboring supercells is $7 \AA$ or more. Both homopairs (like enantiomers) and heteropairs (unlike enantiomers) were considered in each of three potential interaction schemes: internal $\mathrm{OH} / \mathrm{OH}$ interaction, internal $\mathrm{OH} / \mathrm{H}$ interaction, and internal $\mathrm{H} / \mathrm{H}$ interaction. A complete data table for the calculated pairing enthalpies is shown in the Supporting Information. For each relaxed structure the number and calculated length of reasonable intermolecular hydrogen bonds was determined along with the total pairing energy (relative to two molecules at infinite separation). For this study, an $\mathrm{H} \cdots \mathrm{O}$ interaction was considered to be a probable intermolecular 

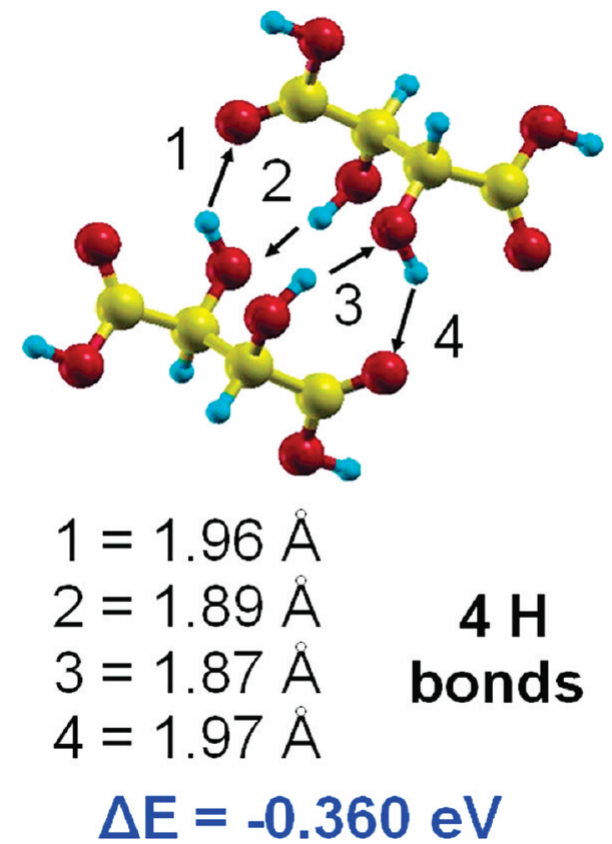

Figure 6. Density functional theory results describing the geometry of the most energetically favorable gas-phase $(S, S)$-tartaric acid pairing interaction. Hydrogen bonding between two opposite enantiomers involves each internal hydroxyl group. The complete spectrum of pairing calculations is shown in the Supporting Information.

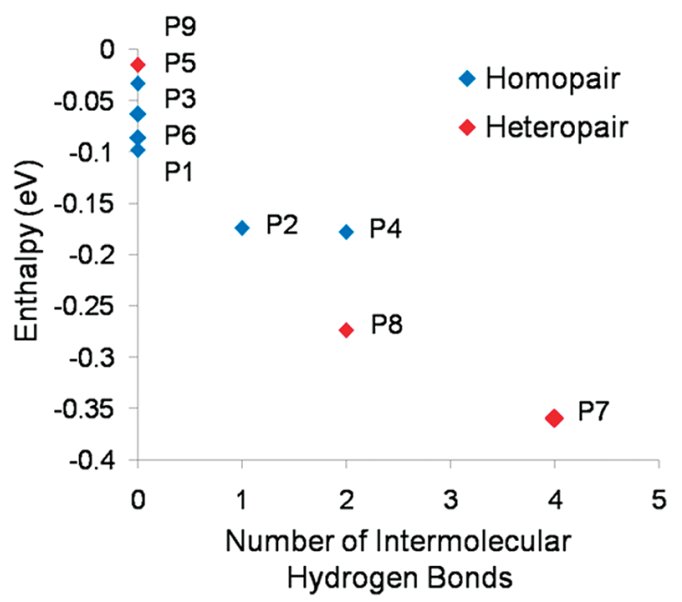

Figure 7. Density functional theory results showing the total enthalpy of pair formation versus number of hydrogen bonds formed for all gasphase tartaric acid pairs. Each data point is the energy associated with a different pair configuration as shown in Figure S4 in the Supporting Information. The most energetically favorable pairing interaction is between two opposite enantiomers where hydrogen bonding involves internal hydroxyl groups as shown in Figure 6 (P7).

hydrogen bond if the distance between the two atoms was $2 \AA$ or less. For cases where no intermolecular hydrogen bonds were formed the intermolecular distance corresponds to the shortest distance measured between the two molecules (but not necessarily $\mathrm{O} \cdots \mathrm{H}$ distances). Upon the formation of four new intermolecular hydrogen bonds, the most energetically favorable structure is the $(R, R)-/(S, S)$-tartaric acid heteropair shown in Figure 6.

A plot of the total energy versus number of hydrogen bonds formed for the previously described gas-phase tartaric acid pairs (P1 through P9 corresponding to the data table and structures presented in the Supporting Information) is shown in Figure 7. The general trend is that the pair binding energy increases (i.e., becomes more exothermic) proportionally as the number of intermolecular hydrogen bonds per pair increases. Attempts were made to elucidate energetic trends based upon other criteria, such as the formation of closed intermolecular rings, but no obvious correlations could be identified. For instance, P2 forms a single intermolecular hydrogen bond, while the structure described by $\mathrm{P} 4$ contains two hydrogen bonds that can be visualized as forming a ring structure. The path of the ring structure is as follows: $\cdots \mathrm{H}-\mathrm{O}-\mathrm{C}-\mathrm{C}-\mathrm{O} \cdot \cdot \mathrm{H}-\mathrm{O}-\mathrm{C}-\mathrm{C}-\mathrm{O} \cdots$, where the terminal $\mathrm{H}$ and $\mathrm{O}$ atoms in this chain are also interacting via a hydrogen bond with one another. Because the final energies of P2 and P4 are identical, no stabilization via ring formation can be deduced.

The most exothermic structure that results from the calculations is the $(R, R) /(S, S)$-tartaric acid heteropair oriented with the internal hydroxyl groups in such a way that four intermolecular hydrogen bonds are formed. The binding energy of this pair corresponds roughly to what is expected for the formation of four new hydrogen bonds (about $-0.1 \mathrm{eV}$ per $\mathrm{H} \cdots \mathrm{O}$ interaction). Therefore, it can be concluded that the molecular basis unit for the racemic films is indeed composed of a pair of opposite tartaric acid enantiomers. This finding is corroborated by both the enantiopairing that takes place in three-dimensional DL-tartaric acid crystals and the lack of separate enantiopure domains in the STM images.

\section{Conclusions}

A comparison of dual component tartaric acid films on $\mathrm{Ag}(111)$ that were formed from two different growth procedures has been made. Low-temperature scanning tunneling microscopy has shown that the long-range film organization resulting from the two different deposition procedures is dictated by intermolecular hydrogen bonding in the limit of a weak interaction with the surface. For enantiopure films, the chirality of the tartaric acid molecule is transferred to global film chirality despite the lack of a rigid and well-defined adsorption geometry. Exposure of the $\mathrm{Ag}(111)$ surface to a racemic tartaric acid mixture does not result in the formation of homochiral conglomerates; in contrast, the two-dimensional films reflect the true racemic nature of the three-dimensional solid. Density functional theory confirms that the racemic films are composed of a new heteropair basis, an effect that results from the enhanced affinity between opposite handed tartaric acid enantiomers.

Acknowledgment. The authors thank Tyler J. Grassman and Sujata Paul for helpful discussions regarding the computational work. Support from the Army Research Office and the Defense Threat Reduction Agency, grant no. W911NF-06-1-0266, is gratefully acknowledged. Supercomputing resources were provided by the National Center for Computational Sciences at Oak Ridge National Laboratory and the High Performance Computing Center at North Carolina State University. This work has also been supported in part by BES, U.S. DOE at ORNL (DEFG02-98ER14847 and DE-AC05-00OR22725 with UT-Batelle, LLC.

Supporting Information Available: Experimental data showing the formation of multilayered tartaric acid structures as well as further density functional theory results that support statements made in the text. This material is available free of charge via the Internet at http://pubs.acs.org.

\section{References and Notes}

(1) Barlow, S. M.; Raval, R. Surf. Sci. Rep. 2003, 50, 201. 
(2) Lorenzo, M. O.; Baddeley, C. J.; Muryn, C.; Raval, R. Nature 2000, 404,376 .

(3) Jones, T. E.; Baddeley, C. J. Surf. Sci. 2002, 513, 453.

(4) Zhao, X. Y.; Zhao, R. G.; Yang, W. S. Surf. Sci. 1999, 442, L995.

(5) Kühnle, A.; Linderoth, T. R.; Hammer, B.; Besenbacher, F. Nature 2002, 415, 891 .

(6) Kühnle, A.; Linderoth, T. R.; Schunack, M.; Besenbacher, F. Langmuir 2006, 22, 2156. 233.

(7) Egawa, C.; Iwai, H.; Kabutoya, M.; Oki, S. Surf. Sci. 2003, 532,

(8) Chen, Q.; Lee, C. W.; Frankel, D. J.; Richardson, N. V. Phys. Chem. Commun. 1999, 2, 41.

(9) Mamdouh, W.; Uji-i, H.; Gesquiere, A.; De Feyter, S.; Amabilino, D. B.; Abdel-Mottaleb, M. M. S.; Veciana, J.; De Schryver, F. C. Langmuir 2004, 20, 9628.

(10) Rankin, R. B.; Sholl, D. S. J. Phys. Chem. B 2005, 109, 16764.

(11) Haq, S.; Massey, A.; Moslemzadeh, N.; Robin, A.; Barlow, S. M.; Raval, R. Langmuir 2007, 23, 10694.

(12) De Feyter, S.; Gesquiere, A.; Wurst, K.; Amabilino, D. B.; Veciana,

J.; De Schryver, F. C. Angew. Chem., Int. Ed. 2001, 40, 3217.

(13) Cai, Y.; Bernasek, S. L. J. Am. Chem. Soc. 2003, 125, 1655.

(14) Cai, Y.; Bernasek, S. L. J. Phys. Chem. B 2005, 109, 4514.

(15) Huang, T.; Hu, Z.; Zhao, A.; Wang, H.; Wang, B.; Yang, J.; Hou,

J. G. J. Am. Chem. Soc. 2007, 129, 3857.

(16) Cortes, R.; Mascaraque, A.; Schmidt-Weber, P.; Dil, H.; Kampen,

T. U.; Horn, K. Nano Lett. 2008, 8, 4162.

(17) Yan, H. J.; Wang, D.; Han, M. J.; Wan, L. J.; Bai, C. L. Langmuir 2004, 20, 7360 .

1.

(18) Humblot, V.; Barlow, S. M.; Raval, R. Prog. Surf. Sci. 2004, 76,

(19) Hermse, C. G. M.; van Bavel, A. P.; Jansen, A. P. J.; Barbosa, L. A. M. M.; Sautet, P.; van Santen, R. A. J. Phys. Chem. B 2004, 108, 11035 . 6639.

(20) Barbosa, L. A. M. M.; Sautet, P. J. Am. Chem. Soc. 2001, 123,

(21) Zhang, J.; Lu, T.; Jiang, C.; Zou, J.; Cao, F.; Chen, Y. J. Chem. Phys. 200 9, 131, 144703.

(22) Haq, S.; Liu, N.; Humblot, V.; Jansen, A. P. J.; Raval, R. Nature Chem. 2009, 1, 409 .
(23) Romer, S.; Behzadi, B.; Fasel, R.; Ernst, K. H. Chem-Eur. J. 2005, 11, 4149.

(24) Astbury, W. T. Proc. R. Soc. London, Ser. A 1923, 104, 219.

(25) Jacques, J.; Collet, A.; Wilen, S. H. Enantiomers, Racemates, and Resolutions; Krieger Publishing Co.: Malabar, FL, 1994.

(26) Lakhani, A. M.; DeWitt, D. J.; Sant'Agata, N. M.; Pearl, T. P. J. Phys. Chem. C 2007, 111, 5750.

(27) Santagata, N. M.; Luo, P.; Lakhani, A. M.; DeWitt, D. J.; Day,

B. S.; Norton, M. L.; Pearl, T. P. IEEE Sens. 2008, 8, 758.

(28) Santagata, N. M.; Lakhani, A. M.; DeWitt, D. J.; Luo, P.; Pearl,

T. P. J. Phys.: Conf. Ser. 2008, 100, 052066.

(29) Lakhani, A. M.; Kelly, S. J.; Pearl, T. P. Rev. Sci. Instrum. 2006, 77, 043709.

(30) Horcas, I.; Fernández, R.; Gómez-Rodríguez, J. M.; Colchero, J.; Gómez-Herrero, J.; Baro, A. M. Rev. Sci. Instrum. 2007, 78, 013705.

(31) Baroni, S.; Dal Corso, A.; De Gironcoli, S.; Giannozi, P., http:// www.pwscf.org/.

(32) Soares, E. A.; Nascimento, V. B.; de Carvalho, V. E.; de Castilho, C. M. C.; de Carvalho, A. V.; Toomes, R.; Woodruff, D. P. Surf. Sci. 1999, $419,89$.

(33) Stern, F.; Beevers, C. A. Acta Crystallogr. 1950, 3, 341.

(34) Okaya, Y. Acta Crystallogr. 1966, 21, 237.

(35) Polavarapu, P. L.; Ewig, C. S.; Chandramouly, T. J. Am. Chem. Soc. 1987, 109, 7382.

(36) Kokalj, A. Comput. Mater. Sci. 2003, 28, 155.

(37) Santagata, N. M. Ph.D. Thesis, North Carolina State University, 2009.

(38) Stabel, A.; Heinz, R.; Rabe, J. P.; Wegner, G.; De Schryver, F. C.; Corens, D.; Dehaen, W.; Sueling, C. J. Phys. Chem. 1995, 99, 8690.

(39) Fasel, R.; Parschau, M.; Ernst, K.-H. Nature 2006, 439, 449.

(40) Rankin, R. B.; Sholl, D. S. Surf. Sci. 2005, 574, L1.

(41) Rankin, R. B.; Sholl, D. S. J. Phys. Chem. B 2005, 109, 16764.

(42) Kohn, W.; Meir, Y.; Makarov, D. E. Phys. Rev. Lett. 1998, 80, 4153.

(43) Grassman, T. J.; Bishop, S. R.; Kummel, A. C. Surf. Sci. 2008, $602,2373$.

(44) Paier, J.; Hirschl, R.; Marsman, M.; Kresse, G. J. Chem. Phys. 2005, 122, 234102.

JP912124V 